\title{
PARTICLE-ROTOR MODEL DESCRIPTION OF DOUBLY ODD Ta ISOTOPES
}

\author{
N. ITACO, L. CORAGGIO, A. COVELLO AND A. GARGANO \\ Dipartimento di Scienze Fisiche, Università di Napoli Federico II \\ and Istituto Nazionale di Fisica Nucleare \\ Complesso Universitario di Monte S. Angelo, Via Cintia, 80126 Napoli, Italy
}

\begin{abstract}
The doubly odd Ta isotopes are studied within the framework of the particle-rotor model. The main aim of this study is to obtain information on the neutron-proton interaction in deformed nuclei, with particular attention focused on the role of the tensor force. To this end, both zero-range and finite-range phenomenological interactions are considered. Comparison of the calculated results with experimental data provides evidence of the importance of the tensor-force effects. We have also performed calculations using a realistic two-body $G$ matrix for the neutron-proton interaction. Some preliminary results are presented here.
\end{abstract}

\section{Introduction}

The neutron-proton interaction plays an important role in the description of doubly odd deformed nuclei. As is well known, the two most important effects associated with the residual interaction between the gdd neutron and the odd proton are the Gallagher-Moszkowski (GM) splitting and the Newby (N) shift. 3 Further information on the neutron-proton interaction may be obtained by studying the odd-even staggering in $K \neq 0$ bands. In fact, this effect may be traced to direct Coriolis coupling of $K \neq 0$ bands with one or more N-shifted $K=0$ bands. Bands which exhibit odd-even staggering represent therefore a significant source of knowledge of the effective neutronproton interaction.

In previous works, ${ }^{3-5}$ we have studied the doubly odd isotopes ${ }^{174,176} \mathrm{Lu}$ within the framework of the particle-rotor model. Our aim was to assess the role of the effective neutron-proton interaction, with particular attention focused on the tensor force. The results of these calculations showed that the tensor force as well as the space-exchange and spin-spin space-exchange force play an important role in the description of $K=0$ bands and of some $K \neq 0$ bands.

Motivated by these findings, we have extended our study to other doubly odd deformed nuclei in the rare-earth region. Here, we present some results concerning the two heavier nuclei ${ }^{180,182} \mathrm{Ta}$. As we shall see in Sec. 3, the experimental data are very well reproduced when using the central plus tensor force proposed long ago by Boisson et al. 6 This is in complete agreement with

procortona: submitted to World Scientific on October 30, 2018 
the results of our previous studies, ${ }^{3-5}$ confirming that this effective neutronproton interaction is quite suitable for the unified model description of doubly odd rare-earth nuclei. Being completely phenomenological in nature, this interaction is of course in no way related to the free nucleon-nucleon $(N N)$ potential.

Over the past several years, realistic effective interactions derived from a free $N N$ potential have been successfully used in the shell-model description of a number of near-magic nuclei. $1 \mathrm{~A}$ To our knowledge, no attempt has instead been made to relate the effective neutron-proton interaction in deformed nuclei to the free $N N$ interaction.

In this situation, we have found it challenging to turn our attention to this problem. To start with, we have used as effective neutron-proton-interaction the bare $G$ matrix derived from the Bonn-A free $N N$ potential. 1 In Sec. 4 we shall present some results of these preliminary calculations which provide considerable encouragement for further work in this direction.

\section{Outline of the Model and Calculations}

We assume that the unpaired neutron and proton are strongly coupled to an axially symmetric core and interact through an effective interaction. The total Hamiltonian is written as

$$
H=H_{0}+H_{\mathrm{RPC}}+H_{\mathrm{ppc}}+V_{n p} .
$$

The term $H_{0}$ includes the rotational energy of the whole system, the deformed, axially symmetric field for the neutron and proton, and the intrinsic contribution from the rotational degrees of freedom. It reads

$$
H_{0}=\frac{\hbar^{2}}{2 \mathcal{J}}\left(\boldsymbol{I}^{2}-I_{3}^{2}\right)+H_{n}+H_{p}+\frac{\hbar^{2}}{2 \mathcal{J}}\left[\left(\boldsymbol{j}_{n}^{2}-j_{n 3}^{2}\right)+\left(\boldsymbol{j}_{p}^{2}-j_{p 3}^{2}\right)\right] .
$$

The two terms $H_{\mathrm{RPC}}$ and $H_{\mathrm{ppc}}$ in Eq. (1) stand for the Coriolis coupling and the coupling of particle degrees of freedom through the rotational motion, respectively. Their explicit expressions are

$$
\begin{gathered}
H_{\mathrm{RPC}}=-\frac{\hbar^{2}}{2 \mathcal{J}}\left(I^{+} J^{-}+I^{-} J^{+}\right), \\
H_{\mathrm{ppc}}=\frac{\hbar^{2}}{2 \mathcal{J}}\left(j_{n}^{+} j_{p}^{-}+j_{n}^{-} j_{p}^{+}\right) .
\end{gathered}
$$

The effective neutron-proton interaction has the general form

$$
V_{n p}=V(r)\left[u_{0}+u_{1} \boldsymbol{\sigma}_{p} \cdot \boldsymbol{\sigma}_{n}+u_{2} P_{M}+u_{3} P_{M} \boldsymbol{\sigma}_{p} \cdot \boldsymbol{\sigma}_{n}+V_{T} S_{12}+V_{T M} P_{M} S_{12}\right],
$$


with standard notation 6 In our calculations we have used a finite-range force with a radial dependence $V(r)$ of the Gaussian form

$$
V(r)=\exp \left(-r^{2} / r_{0}^{2}\right)
$$

as well as a zero-range force,

$$
V_{n p}^{\delta}=\delta(r)\left[v_{0}+v_{1} \boldsymbol{\sigma}_{p} \cdot \boldsymbol{\sigma}_{n}\right] .
$$

As basis states we use the eigenfunctions of $H_{0}$, which, according to the assumed axial and reflection symmetry, take the form

$$
\begin{aligned}
\left|\nu_{n} \Omega_{n} \nu_{p} \Omega_{p} I M K\right\rangle= & \left(\frac{2 I+1}{16 \pi^{2}}\right)^{\frac{1}{2}}\left[D_{M K}^{I}\left|\nu_{n} \Omega_{n}\right\rangle\left|\nu_{p} \Omega_{p}\right\rangle\right. \\
& \left.+(-)^{I+K} D_{M-K}^{I}\left|\nu_{n} \bar{\Omega}_{n}\right\rangle\left|\nu_{p} \bar{\Omega}_{p}\right\rangle\right],
\end{aligned}
$$

where the state $|\nu \bar{\Omega}\rangle$ is the time-reversal partner of $|\nu \Omega\rangle$. We have used the standard Nilsson potential 10 to generate the single-particle Hamiltonians $H_{n}$ and $H_{p}$. The parameters $\mu$ and $\kappa$ have been fixed by using the mass-dependent formulas of Ref. 11. The deformation parameter $\beta_{2}$ has been deduced for each doubly odd isotope from the neighboring even-even nucleus while the singleparticle energies for the odd proton and the odd neutron and the rotational parameter $\frac{\hbar^{2}}{2 \mathcal{J}}$ have been derived from the experimental spectra of the two neighboring odd-mass nuclei.

For the neutron-proton interaction we have used both a finite-range force with a Gaussian radial shape and a $\delta$ interaction. As regards the former, we have explored the role of the tensor force by performing two different calculations, with and without the tensor terms. In both cases for the parameter of the interaction we have adopted the values determined by Boisson et al. 0 in their study of doubly odd nuclei in the rare-earth region. As regards the $\delta$ force, we have used for the strength of the spin-spin term $v_{1}$ the value -0.20 $\mathrm{MeV}$, which leads to the lowest possible disagreement between theory and experiment. More details about the choice of the parameters of the potentials as well as their explicit values can be found in Ref. 3 .

We shall give an outline of the calculations performed with the $G$ matrix derived from the Bonn-A potential in Sec. 4.

\section{Results and Comparison with Experiment}

In this section we present some selected results of our study of the two isotopes ${ }^{182} \mathrm{Ta}$ and ${ }^{180} \mathrm{Ta}$. More precisely, we consider the lowest $K^{\pi}=0^{-}$band 
in ${ }^{182} \mathrm{Ta}$ and the $K^{\pi}=1^{+}$ground-state band in ${ }^{180} \mathrm{Ta}$. A more complete presentation and discussion of our results will be given in a forthcoming paper.

In Fig. 1 we compare the experimental spectrum 12 of the $K^{\pi}=0^{-}$ $p \frac{7}{2}[404] n \frac{7}{2}[503]$ band in ${ }^{182} \mathrm{Ta}$ with the spectra obtained by using the Gaussian force, with and without tensor terms, and the $\delta$ force. We see that the right level order and an excellent agreement with experiment is obtained when using the Gaussian force with tensor terms. The discrepancy between the calculated and experimental excitation energies is at most about $20 \mathrm{keV}$. This is not the case for the calculations performed with the pure central finite-range interaction and the $\delta$ force. In fact, as shown in Fig. 1, these calculations do not reproduce the right level order. This may be traced back to the different values of the calculated $\mathrm{N}$-shift. They are 28,1 , and $-6 \mathrm{keV}$ in case (a), (b), and (c), respectively, to be compared with the experimental value of $26 \mathrm{keV}$.

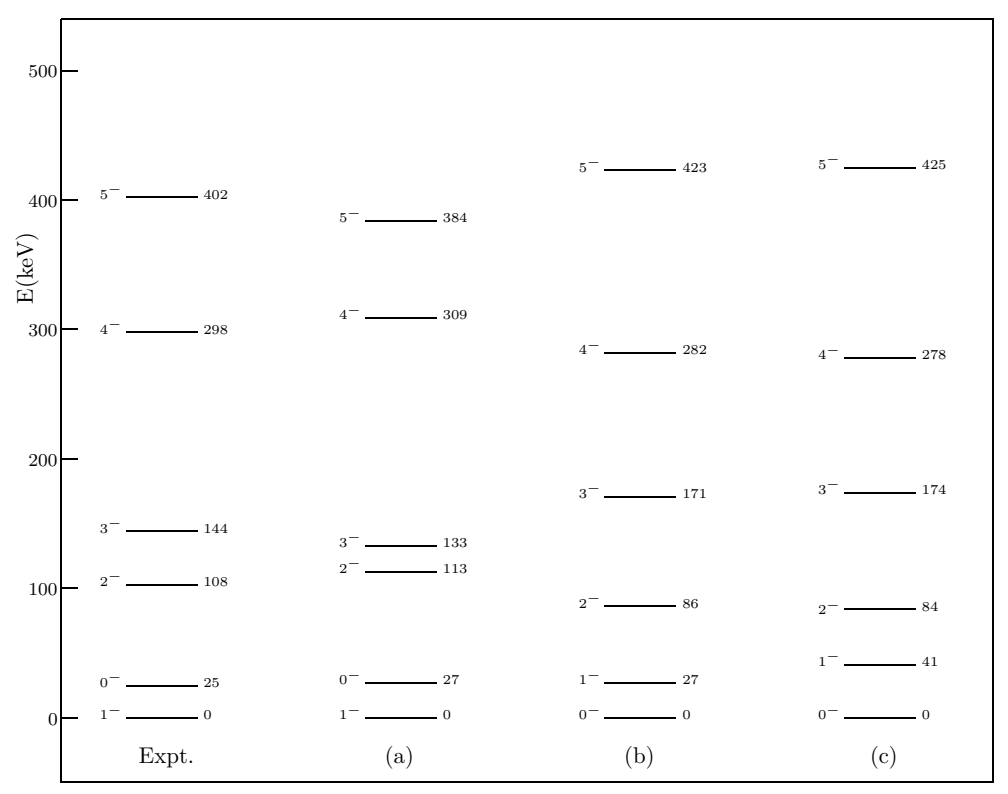

Figure 1. Experimental and calculated spectra of the lowest $K^{\pi}=0^{-}$band in ${ }^{182} \mathrm{Ta}$. The theoretical spectra have been obtained by using (a) a central plus tensor force with a Gaussian radial shape, (b) a Gaussian central force, and (c) a $\delta$ force.

Therefore, while in case (a) the calculated $\mathrm{N}$-shift is in very good agreement with experiment, it becomes too small in case (b), and in case (c) it has even 
the wrong sign.

Let us now come to the $K^{\pi}=1^{+} p \frac{7}{2}[404] n \frac{9}{2}[624]$ ground-state band in ${ }^{180} \mathrm{Ta}$. This band exhibits a rather large odd-even staggering, as can be seen from Fig. 2 where the experimental 13.14 ratio $[E(I)-E(I-1)] / 2 I$ is plotted vs $I$ and compared with the calculated ones.

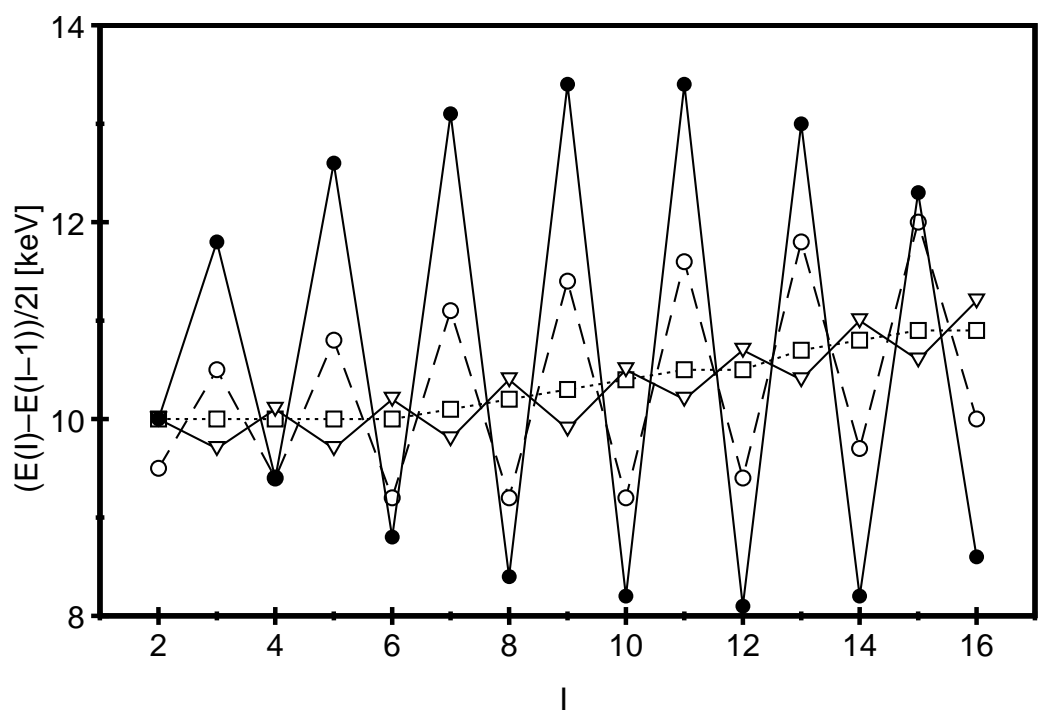

Figure 2. Experimental and calculated odd-even staggering of the $K^{\pi}=1^{+}$ground-state band in ${ }^{180} \mathrm{Ta}$. Solid circles correspond to experimental data. The theoretical results are represented by open circles (Gaussian central plus tensor force), squares (Gaussian central force), and triangles ( $\delta$ force).

We see that the experimental behavior is well reproduced by the calculation including the tensor force. When using the pure central Gaussian force the staggering is almost nonexistent and in the case of the $\delta$ force not only its magnitude is very small, but it has also the opposite phase. It should be pointed out that the staggering in this band may be traced to direct Coriolis coupling with the $K^{\pi}=0^{+} p \frac{7}{2}[404] n \frac{7}{2}[633]$. The fact that only the calculation including the tensor terms gives the right staggering implies therefore that only this force is able to produce a sizeable $\mathrm{N}$ shift for this $K^{\pi}=0^{+}$band. Since this band has not been recognized in ${ }^{180} \mathrm{Ta}$, a direct comparison is not possible at present. It should be noted, however, that the lowest $K^{\pi}=0^{+}$ band observed in ${ }^{174} \mathrm{Lu}$ corresponds just to the same intrinsic n-p configuration. In our study of ${ }^{174} \mathrm{Lu}$ it turned out that only the Gaussian plus tensor 
force is able to produce a spectrum of this band in good agreement with the experimental one.

\section{Calculations with a Realistic Two-Body $G$ Matrix}

As already mentioned in the Introduction, we present here some results of preliminary calculations performed by using as neutron-proton residual interaction the bare $G$ matrix derived from the Bonn-A free $N N$ potential. $G$ matrix is defined 15 by the integral equation

$$
G(\omega)=V+V Q_{2} \frac{1}{\omega-Q_{2} T Q_{2}} Q_{2} G(\omega),
$$

where $V$ represents the $N N$ potential, $T$ denotes the two-nucleon kinetic energy, and $\omega$ is the so-called starting energy. The operator $Q_{2}$ is the Pauli exclusion operator for two interacting nucleons, and its complement $P_{2}=$ $1-Q_{2}$ defines the space within which the $G$ matrix is calculated. All the states outside the $P_{2}$ space are intermediate states represented by plane wave functions. In spherical shell-model calculations the $P_{2}$ space is defined in terms of harmonic oscillator eigenvectors. For deformed nuclei, we have calculated the $G$ matrix making use of Nilsson basis states. The calculation of the $G$ matrix is performed in an essentially exact way by using the Tsai-Kuo method. 16

In Tables 1 and 2 we report the calculated and experimental GM splittings for ${ }^{182} \mathrm{Ta}$ and ${ }^{180} \mathrm{Ta}$. We see that while all the signs are correctly reproduced

Table 1. Experimental and calculated GM splittings (keV) in ${ }^{182} \mathrm{Ta}$.

\begin{tabular}{|cccccc|}
\hline \multicolumn{2}{|c}{ Configuration } & & & & \\
Proton & Neutron & $K_{<}^{\pi}$ & $K_{>}^{\pi}$ & Expt. & Calc. \\
\hline $7 / 2[404]$ & $1 / 2[510]$ & $3^{-}$ & $4^{-}$ & -100 & -172 \\
$7 / 2[404]$ & $3 / 2[512]$ & $2^{-}$ & $5^{-}$ & 139 & 374 \\
$7 / 2[404]$ & $7 / 2[503]$ & $0^{-}$ & $7^{-}$ & -121 & -146 \\
$9 / 2[514]$ & $1 / 2[510]$ & $4^{+}$ & $5^{+}$ & 148 & 149 \\
$9 / 2[514]$ & $3 / 2[512]$ & $3^{+}$ & $6^{+}$ & -98 & -105 \\
$5 / 2[402]$ & $1 / 2[510]$ & $2^{-}$ & $3^{-}$ & 114 & 84 \\
& & & & & \\
\hline
\end{tabular}

also the quantitative agreement can be considered satisfactory, the largest discrepancy with experiment being $100 \mathrm{keV}$ in one case only. As regards the

procortona: submitted to World Scientific on October 30, 2018 
$\mathrm{N}$ shift, we obtain $46 \mathrm{keV}$ for the the lowest $K^{\pi}=0^{-}$band in ${ }^{182} \mathrm{Ta}$, to be compared with the experimental value of $26 \mathrm{keV}$.

Table 2. Same as Table 1, but for ${ }^{180} \mathrm{Ta}$.

\begin{tabular}{|cccccc|}
\hline \multicolumn{2}{|c}{ Configuration } & & & & \\
Proton & Neutron & $K_{<}^{\pi}$ & $K_{>}^{\pi}$ & Expt. & Calc. \\
\hline $7 / 2[404]$ & $9 / 2[624]$ & $1^{+}$ & $8^{+}$ & -100 & -186 \\
$7 / 2[404]$ & $5 / 2[512]$ & $1^{-}$ & $6^{-}$ & -88 & -78 \\
& & & & & \\
\hline
\end{tabular}

\section{Summary}

In this paper, we have presented some results of a particle-rotor model study of doubly odd $\mathrm{Ta}$ isotopes aimed at obtaining detailed information on the effective neutron-proton interaction in the rare-earth region. In particular, we have explored the role of tensor force, which has been neglected in most of the existing studies to date. A comparison of our results with experimental data evidences the importance of this force for the description of $K=0$ bands and of some $K \neq 0$ bands, thus confirming the results of our previous study of Lu isotopes. ${ }^{3-5}$

As regards the results obtained by using a realistic two-body $G$-matrix interaction, which does not contain any adjustable parameter, they are certainly better than what one would expect. This shows that effective interactions derived from the free $N N$ potential may be suitable for use in the unified model description of deformed nuclei. Further work in this direction is in progress.

\section{Acknowledgments}

This work was supported in part by the Italian Ministero dell'Università e della Ricerca Scientifica e Tecnologica (MURST). NI thanks the European Social Fund for financial support.

\section{References}

1. C. J. Gallagher and S. A. Moszkowski, Phys. Rev. 111, 1282 (1958).

2. N. D. Newby, Jr., Phys. Rev. 125, 2063 (1962).

3. A. Covello, A. Gargano, and N. Itaco, Phys. Rev. C 56, 3092 (1997). 
4. N. Itaco, A. Covello, and A. Gargano, in Highlights of Modern Nuclear Structure, ed. A. Covello, (World Scientific, Singapore, 1999), p. 451.

5. N. Itaco, A. Covello, and A. Gargano, in Perspectives on Theoretical Nuclear Physics, Proceedings del Settimo Convegno su Problemi di Fisica Nucleare Teorica, Cortona, 1998, (ETS Editrice, Pisa, 1999), p. 287.

6. J. P. Boisson, R. Piepenbring, and W. Ogle, Phys. Rep. 26, 99 (1976).

7. L. Coraggio, A. Covello, A. Gargano, N. Itaco, and T. T. S. Kuo, J. Phys. G 26, 1697 (2000), and references therein.

8. A. Covello, L. Coraggio, A. Gargano, and N. Itaco, Acta Phys. Pol. B, (2001), in press, and references therein.

9. R. Machleidt, K. Holinde, and Ch. Elster, Phys. Rep. 149, 1 (1987).

10. C. Gustafson, I. L. Lamm, B. Nilsson, and S. G. Nilsson, Ark. Fys. 36, 613 (1967).

11. S. G. Nilsson, C. F. Tsang, A. Sobiczewski, Z. Szymánski, S. Wycech, C. Gustafson, I. -L. Lamm, P. Möller, and B. Nilsson, Nucl. Phys. A 131, 1 (1969).

12. NNDC On-Line Data Service from ENSDF database.

13. G. D. Dracoulis, S. M. Mullins, A. P. Byrne, F. G. Kondev, T. Kibédi, S. Bayer, G. J. Lane, T. R. McGoram, and P. M. Davidson, Phis. Rev. C 58, 1444 (1998).

14. T. R. Saitoh et al., Nucl. Phys. A 660, 121 (1999).

15. E. M. Krenciglowa, C. L. Kung, T. T. S. Kuo, and E. Osnes, Ann. Phys. (N.Y.) 101, 154 (1976).

16. S. F. Tsai and T. T. S. Kuo, Phys. Lett. B 39, 427 (1972). 